\title{
Frontières
}

\section{Les peurs ont-elles une histoire ?}

Extraits d'une entrevue avec Jean Delumeau réalisée par André Champagne

\section{Jean Delumeau et André Champagne}

Volume 12, numéro 2, printemps 2000

Peur bleue...

URI : https://id.erudit.org/iderudit/1074395ar

DOI : https://doi.org/10.7202/1074395ar

Aller au sommaire du numéro

Éditeur(s)

Université du Québec à Montréal

ISSN

1180-3479 (imprimé)

1916-0976 (numérique)

Découvrir la revue

Citer ce document

Delumeau, J. \& Champagne, A. (2000). Les peurs ont-elles une histoire ? Extraits d'une entrevue avec Jean Delumeau réalisée par André Champagne. Frontières, 12(2), 28-34. https://doi.org/10.7202/1074395ar d'utilisation que vous pouvez consulter en ligne.

https://apropos.erudit.org/fr/usagers/politique-dutilisation/ 


\section{LES PEURS ONT-ELLES UNE HISTOIRE?}

\section{Extraits d'une entrevue ${ }^{1}$ avec Jean Delumeau réalisée par André Champagne}

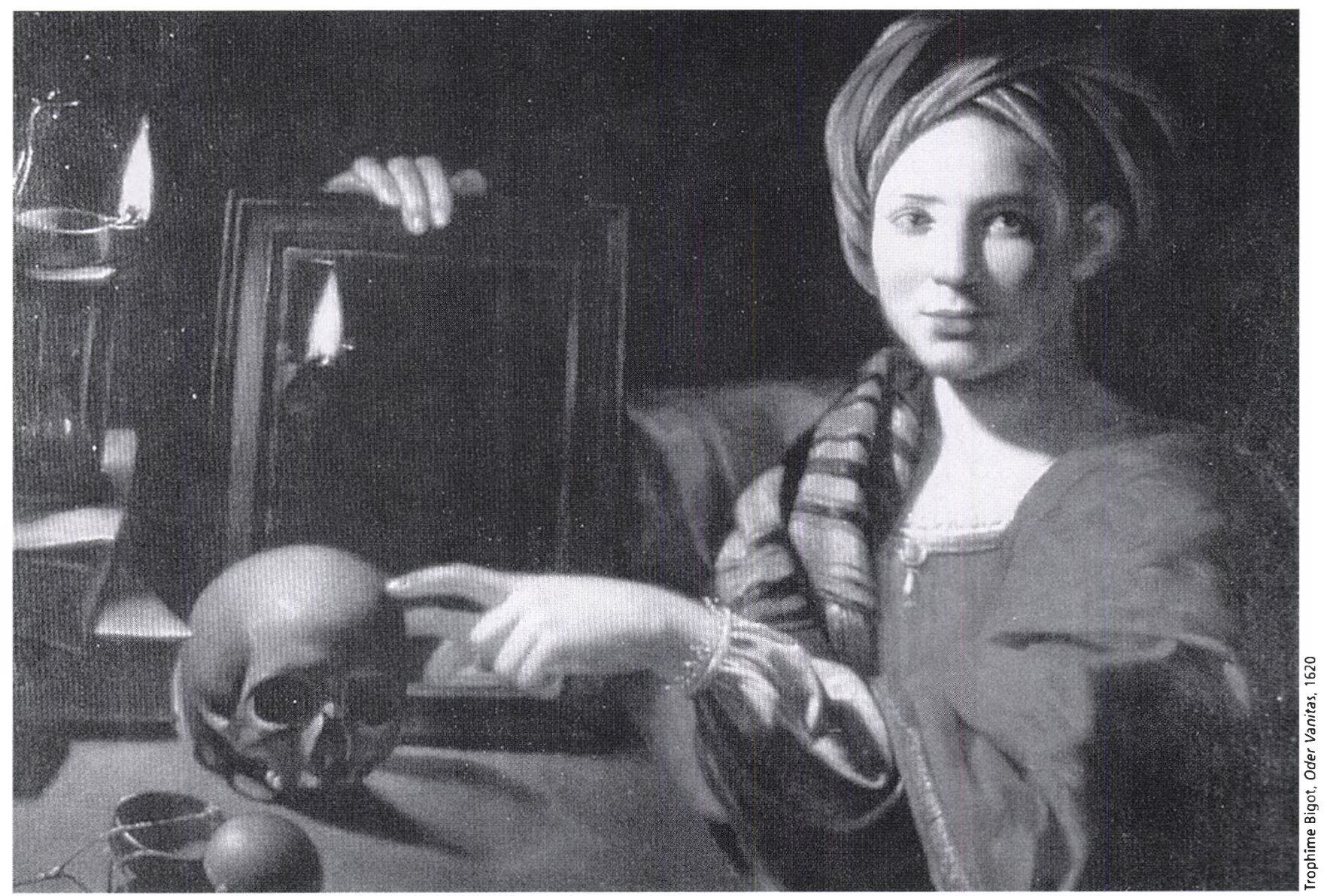

Jean Delumeau,

historien des religions et professeur honoraire au Collège de France.

\section{André Champagne,}

professeur d'histoire au Collège Jean-de-Brébeuf et chroniqueur à la Société Radio-Canada.

\section{DES SENTIMENTS SI BIEN PARTAGÉS}

\section{Pourquoi une histoire de la peur?}

Il y a deux raisons. La première, c'est que ce sujet n'avait pas été traité et il faut bien voir que l'historien fait de l'histoire comme le musicien fait de la musique. C'est-à-dire que, s'il y a un sujet qui est resté à peu près vierge, il est tenté de vouloir le traiter. C'est ce que j'ai fait, et je ne m'en repens pas; car il y avait là un très vaste secteur à défricher. Si l'on veut faire - ce que l'on tente actuellement à l'échelle mondiale - une histoire des sentiments et des comportements humains, il est paradoxal de laisser de côté des sentiments tels que la peur ou le sentiment de sécurité ${ }^{2}$.

Il y avait donc là, "un créneau», comme on dit quelquefois, à occuper et je me suis situé à l'intérieur de ce créneau. Mais vous pourrez me dire: "Est-ce que vous n'avez pas ici aussi des raisons personnelles?» Alors c'est vrai, j'en avais. Je les ai d'ailleurs évoquées brièvement dans deux pages autobiographiques qui se trouvent dans l'introduction de La peur en Occident (1978) et qui valent pour l'ensemble de mon oeuvre. J'y explique qu'effectivement j'étais très marqué par la peur dans mon enfance. C'était peut-être une façon, si vous voulez, de l'évacuer, de l'exorciser. En tout cas, quand je me suis mis à travailler sur le thème de la peur, j'ai pensé qu'effectivement des traumatismes de l'enfance m'avaient dirigé vers cette voie. Reste vrai toutefois que le sujet avait été peu étudié par les historiens. 
Comment se manifeste ce sentiment de peur et qui avait peur et peur de quoi?

Tout d'abord, il faut dire que la peur est un sentiment normal. Il ne faut pas avoir peur d'avoir peur, si j'ose dire, ni rougir d'avoir peur. La peur comme conscience d'un danger est une chose et une réaction excessive de peur se traduisant soit par des paniques individuelles ou collectives, soit au contraire par une espèce d'immobilisation durant laquelle on est incapable de réagir en est une autre. $[. .$.

Deuxième point, tout le monde autrefois avait peur de la mer, de l'orage, de la peste. Ainsi quand la peste sévissait dans une ville, c'était vraiment un traumatisme incroyable. À notre époque, nous avons peur du sida et nous avons raison. Mais la peste, c'était bien pire, parce qu'une peste enlevait en trois mois la moitié de la population d'une ville. Par conséquent, quand la peste se déclarait dans une ville, il était tout à fait légitime d'avoir peur et tout le monde avait peur. Il y en a qui surmontaient leur peur et d'autres qui ne la surmontaient pas, mais tout le monde avait peur. Ainsi certaines peurs étaient partagées par tout le monde de la même façon. Supposez, ce qu'à Dieu ne plaise, qu'éclate une conflagration nucléaire, tout le monde aurait peur et ce serait normal. Quelques-uns sans doute conserveraient leur sang-froid. Mais la plupart ne le conserveraient pas! Dans toute civilisation, il y a des dangers tels que tout le monde en a légitimement peur.

En revanche tout le monde n'avait pas peur des chats, mais Ronsard avait peur des chats en qui il voyait des animaux démoniaques. La plupart des gens avaient peur des hérétiques, mais tout le monde n'avait pas peur des hérétiques et il s'est trouvé des marginaux, il faut bien le dire, du christianisme pour dire: "ne condamnons pas les hérétiques, l'hérétique n'est pas un démon». 11 y avait donc des peurs qui frappaient tout le monde et d'autres au contraire qui étaient plus spécifiques.

\section{Pourquoi cette peur du passé?}

Eh bien, il faut voir qu'autrefois on vivait dans une civilisation très largement animiste et vitaliste, c'est-àdire que l'on n'apercevait pas de frontières nettes entre un domaine matériel et un domaine spirituel. Ils étaient véritablement imbriqués l'un dans l'autre. Et on ne voyait pas non plus, beaucoup moins que de nos jours en tout cas, de coupures nettes entre la vie et la mort. Ce qui fait qu'il y avait dans la mentalité de beaucoup de gens ce qu'Edgar Morin et d'autres ont appelé «la survie du double». La survie du double, cela veut dire que les morts, croyait-on, continuaient de quelque façon à vivre dans le monde des vivants et vivaient d'une façon, je dirais, atténuée: ils avaient un corps impalpable, mais que parfois l'on pouvait voir, d'où la tenace croyance dans les revenants.

Autrefois, on a largement cru aux revenants, parce que précisément on ne se faisait pas de la mort la même idée que nous. Les morts continuaient de quelque façon à venir dans les maisons, dans les champs où ils avaient vécu et, en certaines nuits, notamment la nuit de la Toussaint, ils se rassemblaient dans des lieux déterminés. La croyance aux revenants a été si forte qu'un juge d'Angers à la fin du XVIe siècle put exposer le cas suivant: quelqu'un loue une maison; il se trouve que cette maison est hantée, il se plaint au propriétaire et il refuse de payer le loyer. Le juriste reconnaît qu'il a raison de ne pas payer le loyer d'une maison qui est hantée. Voilà un cas concret, n'est-ce pas, qui nous prouve que cette croyance était très largement répandue $^{3}[\ldots]$

\section{CONSTRUCTIONS MENTALES 4}

Le mépris du monde et la pastorale de la peur sont-ils une réaction du monde chrétien face à la menace constante d'hérésies, à partir du XIe siècle?

Je ne le crois pas. Le mépris du monde part d'une origine différente. Le mépris du monde s'enracine, à mon avis, dans la philosophie grecque. Le mépris du monde ne vient pas du tout du judaïsme. Le judaïsme n'a jamais méprisé le monde. Au contraire. Dans le monde hébreu, on ne faisait pas du tout l'éloge de la virginité. C'est la vie sexuelle familiale qui constituait la vie normale. Le mépris du monde vient de la philosophie néo-platonicienne, qui considérait que le malheur existentiel, au sens strict du terme, c'était la chute de l'âme dans le corps. Il fallait donc essayer de se détacher au maximum du corps pour redonner à l'âme sa liberté. [...]

Mais il est vrai que la doctrine du mépris du monde a été intégrée au christianisme. Et comment l'a-t-elle été? Elle l'a été par les moines. Car cette doctrine sur laquelle j'ai longuement insisté dans mon livre Le péché et la peur n'était destinée qu'aux moines. Elle a été élaborée par les moines et à l'usage des moines. Voilà des gens qui, pour approcher Dieu de plus près, se séparaient du monde, c'était la fuga Mundus, la "fuite du monde». Comme c'était un choix difficile, ils s'y exhortaient, ce qui est normal, par une doctrine du mépris des choses qu'ils quittaient. Ils s'exhortaient à quitter le monde en jetant l'anathème sur lui.

Il faut bien voir que cette doctrine n'était pas destinée au grand public. Elle était, à l'origine, destinée à des ascètes, donc à une toute petite minorité qui avait besoin de se conforter dans son propre choix. Mais ce qui a été, et je n'hésite pas à le dire, un des drames de l'histoire chrétienne, c'est que la prédication, surtout à partir du XIIIe siècle et notamment par les Ordres mendiants, a répandu à l'usage d'une civilisation entière ce qui était une doctrine qui n'aurait dû être à l'usage que de quelques-uns. [...]

$D u$ contemptus mundi, du mépris du monde, les moines sont passés à la culpabilisation. Comment celleci est-elle présentée aux fidèles?

Je ne parlerai ici que de 1'Occident, parce que les choses ne se sont pas passées de la même façon dans la chrétienté orientale. La culpabilisation en Occident a été dramatisée, on peut le dire, à partir de saint Augustin. Celui-ci a fait une sorte de fixation sur le péché originel, l'expression d'ailleurs est de lui. Car, dans les Évangiles, il n'est jamais question du péché originel. Il est seulement question du "péché du monde». Jésus n'a pas soufflé mot d'Adam et Ève. Il n'a pas soufflé mot d'une culpabilité héréditaire. Elle est en revanche présente dans saint Paul et bien plus encore dans les livres de saint Augustin qui, pour faire bonne mesure, y a ajouté la doctrine de «la masse de perdition». Ce qui veut dire que la majorité des gens sera damnée et qu'il n'y aura qu'une minorité d'élus, grâce à la rédemption; sinon, personne n'aurait été sauvé. 
Voilà la doctrine traditionnelle sur laquelle on a fonctionné en Occident, et je mets n'importe qui au défi de dire que je me trompe en affirmant cela. Telle a été la doctrine massivement reçue fondée, en outre, sur une mauvaise compréhension de la parole des Évangiles, «beaucoup d'appelés et peu d'élus» qui, en réalité, est simplement un proverbe - un proverbe hébraique pas une doctrine théologique. D'ailleurs, il y a très peu de théologie chez les juifs. Alors vous comprenez qu'à partir de ces éléments il était facile, et avec la plus grande conviction - ce n'était pas de la tactique - de dire aux gens: «Il y aura beaucoup d'appelés et peu d'élus, faites très attention, vous êtes tous pécheurs, vous êtes tous coupables, vous êtes tous menacés d'aller en enfer. Faites très attention.» Quelle doctrine dramatique!

Les choses n'ont pas été exactement semblables dans la chrétienté orientale. Car on y a parlé assurément, en termes historiques, comme chez nous en Occident, du péché d'Adam et Ėve, mais on n'a pas fait une fixation sur ce pêché et on a mis davantage l'accent sur la rédemption universelle. [...]

Dans La peur en Occident, vous consacrez un chapitre aux comportements collectifs en période de peste. Cette tendance qu'avaient les sociétés occidentales à chercher des coupables est étonnante!

Ce n'était pas là seulement un comportement propre aux Occidentaux. Je crois qu'il s'agit d'un comportement humain fondamental. Lorsqu'une catastrophe individuelle ou collective arrive, c'est une tendance assez naturelle de chercher des coupables. Quelquefois on va trouver que le coupable, c'est soi-même. Mais le plus souvent, ce sera les autres. Quand un malheur arrive, c'est une tendance naturelle de chercher qui l'a provoqué, qui est le coupable. Quand survenait une épidémie de peste, qui provoquait vraiment, comme je le disais précédemment, un traumatisme collectif énorme, alors la tendance était encore plus forte.

Il faut dire que l'Église d'une certaine façon invitait à ce regard. Je dois toutefois nuancer mon propos, l'Église présentait en général les pestes comme des punitions. Mais des punitions envoyées par Dieu contre une collectivité coupable. L'Église n'invitait pas à chercher des boucs émissaires autres que soi-même. Mais si l'on entreprenait, par exemple, des pèlerinages ou des processions, c'étaient des processions pénitentielles pour demander pardon pour les péchés de la ville qui avait fauté par des comportements ou trop cupides ou trop lascifs, etc. Une accumulation de péchés avait provoqué la punition des villes. Mais les gens n'entendaient pas toujours très bien ce discours et, plutôt que de s'autoaccuser, ils cherchaient dans la collectivité quelqu'un ou quelques-uns qui avaient attiré la punition sur leur ville. Ces accusés furent les lépreux, les Juifs ou des gens que l'on croyait avoir vus répandre des produits mortifères, des venins et des graisses sur les portes, ce qui provoquait ainsi l'épidémie. [...] [Comme on l'a vu lors de la Peste Noire de 1348-1351]

Qu'entendez-vous par la culture dirigeante de la peur?

J'entends par culture dirigeante, le monde intellectuel, et, plus généralement, le monde de ceux qui avaient la parole et le pouvoir. Voilà! Qui avaient la parole et le pouvoir. Or, à l'époque que j'ai spécialement étudiée, soit entre 1348 et 1648 , les gens qui avaient surtout la parole et le pouvoir qu'elle donne étaient les gens d'Église. C'étaient les gens d'Église parce que, autrefois, le principal des médias était la prédication. Je ne sais pas si nos contemporains saisissent bien [...] quelle était la situation concrète, quand il n'y avait ni journaux, ni radio, ni télévision. Le principal des médias, c'était, je le répète, la prédication, d'où, entre parenthèses, une énorme responsabilité des prédicateurs.

Prenons un cas précis en dehors même des pestes: [...] saint Vincent Férier, prédicateur de la fin du XIVe et des débuts du XVe siècle, a circulé de la Catalogne à la France et à l'Italie, etc., pour mourir finalement à Vannes. C'est quelqu'un qui a eu une audience internationale. Or, nombre de ses sermons constituaient une annonce de la venue de l'Antéchrist et de la fin du monde. $[\ldots]$

\section{Quels phénomènes pouvaient inciter ces gens à avoir peur?}

Je pense que, en cas de malheurs répétés et cumulés, les gens ont tout naturellement tendance, à toute époque, à anticiper un avenir encore plus noir et, par conséquent, à penser que les derniers temps sont arrivés ou vont arriver. Prenons par exemple la période du Grand Schisme, qui a duré de 1378 à 1417, donc 39 ans. Or, vous savez, 39 ans pour une vie d'autrefois, c'était beaucoup - cette période fut vraiment une période de catastrophes. D'une part, la peste était installée, elle était arrivée en 1348. Certes, la principale épidémie était terminée, mais d'autres suivirent de façon récurrente. La peste était là, et pour longtemps. D'autre part, il y avait la guerre de Cent Ans entre la France et l'Angleterre, les ravages de brigands et diverses guerres civiles, que je passe. Et puis il y avait la chrétienté d'Occident coupée en deux parfois, même en trois. Alors, à quel pape devait-on obéir? C'était vraiment un scandale, non seulement pour l'Église dirigeante, mais aussi pour l'ensemble des fidèles. Ajoutez à cela l'avance turque. C'est le moment où les Turcs avancent de façon décisive. D'abord, ils s'approchent de Constantinople qui va être prise en 1453, mais ils progressent de façon dramatique dans les Balkans. Tous ces malheurs réunis firent que les gens eurent l'impression que les derniers temps étaient proches, ou déjà arrivés. N'imaginons pas ces gens-là donnant à l'humanité une longue tranche d'histoire à vivre, pour eux l'histoire humaine approchait de son terme.

\section{Comment les prophéties apocalyptiques sont-elles interprétées?}

Il y a eu deux interprétations des prophéties apocalyptiques mais parfois, elles interférèrent l'une avec l'autre. Selon la conception la plus fréquente - celle de l'Église officielle - on était entré dans la période terminale de l'histoire humaine qui finira par la fin du monde, sans préciser de date. On était entré dans cette phase terminale de l'histoire humaine depuis que le Christ est venu. Mais il y a eu l'autre interprétation de l'Apocalypse de saint Jean, celle qui a mis l'accent sur le millénium. Je m'explique: dans l'Apocalypse de saint Jean, il est dit en substance qu' 'il va y avoir une période de convulsions, puis mille ans durant lesquels le démon sera enchaîné, mille ans de bonheur durant lesquels le Christ régnera sur terre avec les élus, suivront une dernière intrusion démoniaque, une dernière convulsion et la fin du monde». 
Dans le premier schéma dont j'ai parlé il n'est pas question des mille ans. Les mille ans, c'est ce qu'on est en train de vivre avant la fin du monde. Au contraire, dans l'autre schéma, on espère qu'après une traversée difficile on va aboutir à une période très heureuse sur terre avant les dernières convulsions et la fin du monde ${ }^{5}$. Les deux schémas ont été présents l'un à côté de l'autre, mais il faut dire que mon découpage est un peu systématique et que parfois des gens ont pu mêler les deux programmes. [...]

\section{Le monde protestant a-t-il échappé à cette pastorale de la peur?}

Non, on aurait pu penser qu'il y aurait échappé, puisque la doctrine de Luther et de Calvin sur la justification par la foi consistait à dire précisément aux chrétiens: "Nous sommes pécheurs. Nous le resterons toute notre vie. Mais nous sommes déjà pardonnés. Il n'y a même pas de purgatoire.» Par conséquent cette doctrine se voulait rassurante. Mais elle a été doublée par celle de la prédestination aussi présente chez Luther que chez Calvin. Et Luther qui l'a lancée dans son livre sur le Serf arbitre, opposé à la doctrine d'Érasme. S'il y a prédestination, si nous sommes un jouet dans les mains de Dieu, qu'est-ce qui nous prouve que nous sommes vraiment sauvés?

Dès lors l'angoisse était réintroduite dans le discours religieux et les fidèles invités à passer par une séquence "de conversion» pour tenter d'obtenir la certitude qu'ils étaient sauvés. La "pastorale de la peur» était ainsi bel et bien réinjectée dans le monde protestant. La dernière partie de mon livre, Le péché et la peur, est justement consacrée à ce thème en le pays réformé. Mais il faut dire que le luthéranisme et l'anglicanisme ont progressivement amorti la doctrine de la prédestination et, par conséquent, essayé d'éteindre l'angoisse dont je parlais tout à l'heure. Mais cela a été beaucoup moins vrai dans le secteur puritain. [...]

Si la vie sur terre était à ce point pénible, si les occasions de péchés étaient aussi abondantes, comment présentait-on alors l'enfer? [...]

L'enfer était vraiment effrayant et je pense sérieusement que le discours sur l'enfer, pendant une assez longue séquence de l'histoire chrétienne, a eu un véritable impact. L'enfer était décrit comme la somme de tous les supplices. Imaginez les tourments les plus affreux, de toutes catégories, mettez-les tous en enfer, vous restez encore en dessous de la réalité. Tel était le discours qui était tenu sur l'enfer. [...]

Avec un discours aussi tragique, les fidèles devaient sûrement se résigner au pire. Croyaient-ils alors au salut éternel?

Je pense qu'ils y croyaient parce que, tout de même - et c'est là le thème de mon livre Rassurer et protéger - il y avait une contrepartie. Ce discours sur la peur aurait été intenable sans contrepartie. L'historien que je suis se devait de démontrer en quoi elle a consisté. Je ne parle pas ici des remèdes contre les dangers d'ici-bas, mais uniquement des moyens d'échapper aux dangers de l'au-delà.

À cet égard, il y a eu deux grandes réponses chrétiennes en Occident: la catholique et la protestante. La réponse protestante est celle que je vous ai dite précé- demment: "Nous sommes pécheurs mais nous sommes déjà sauvés, à condition de croire à la parole du Sauveur." Si l'on met de côté la prédestination, c'était une réponse qui se voulait rassurante, et Luther luimême s'est rassuré avec cette doctrine.

La doctrine [catholique] a fourni de son côté une réponse que je dirais parallèle et qui a consisté à dire: "Nous sommes pécheurs mais le remède existe: c'est la confession. La confession qui vous remet en état de grâce, donc en état de mériter le paradis chaque fois que nous péchons." Le remède était donc à portée de la main avec les sacrements en général et la confession en particulier. Ainsi l'Église catholique n'a pas laissé les gens dans la misère de leur peur. Elle leur a constamment proposé les moyens d'en sortir et je pense que les fidèles espéraient, tout de même, aller au Paradis. [...]

\section{UN FÉMININ DIABOLISÉ}

Le juif et le musulman "agents de Satan", à la rigueur on pourrait comprendre, mais la femme "agent de Satan", c'est étonnant!

Il faut nuancer. Il y a eu dans le christianisme deux attitudes très différentes vis-à-vis de la femme. Il a nourri incontestablement une attitude et un discours valorisants. Car jamais, je pense, aucune religion n'avait mis une femme aussi haut que la Vierge Marie, sous le vocable duquel se trouve la plupart de nos cathédrales et de nos plus belles églises. Il s'est produit une valorisation de la femme à travers Marie et plus généralement une valorisation de la femme vierge ou, à la rigueur, de la femme veuve. C'est l'aspect positif. La femme mariée, vivant sa vie de ménage habituelle, a toutefois été beaucoup moins exaltée.

L'aspect négatif vient évidemment de ce que le christianisme a été pris en charge surtout par des célibataires et souvent des moines qui étaient donc, eux aussi, des célibataires et qui, évidemment, devaient se défendre peut-être contre la tentation de la chair. De sorte qu'ils ont eu tendance à voir dans la femme l'être par excellence qui risquait de les détourner de leur vocation. À cette défiance on a cherché des causes et on les a trouvées dans le récit de la Genèse, puisque c'est la femme qui tend la pomme à Adam. Ce qu'il faut bien voir, c'est que très longtemps, disons au moins jusqu'au XVIIIe siècle, le récit de la Genèse a été pris au pied de la lettre - cela peut nous étonner - tant du côté catholique que du côté protestant. L'expression de "récit historique" pour caractériser les premiers chapitres de la Genèse, donc la création du monde, l'épisode du paradis terrestre, le premier péché, est commune à saint Augustin, Isidore de Séville, saint Thomas d'Aquin, Luther et on peut ajouter Calvin. Au moins jusqu'au XVIIIe siècle, mis à part des exceptions comme Origène et quelques autres, ce récit a été pris absolument au pied de la lettre. Dans ces conditions, c'est la femme qui, tentée par le serpent, avait induit Adam en tentation et l'avait fait pécher. La femme était responsable comme Pandore dans la mythologie grecque. Pour les chrétiens, Ève était responsable de toutes les catastrophes qui étaient arrivées à l'humanité et on avait donc raison de se méfier de la femme. Voilà les deux langages qui ont été tenus au sujet d'elle: un langage très valorisant et un langage très dévalorisant ${ }^{6}$. 
Établit-on une relation entre la femme et le diable?

Oui, parce que, précisément dans le récit du premier péché, la femme a été l'agent du diable. Or, l'époque dite de la Renaissance a été l'époque de craintes maximales de Satan. Car le grand moment des ouvrages de démonologie n'est pas le Moyen Âge, mais la Renaissance. Tous ces ouvrages mettent en cause la femme, parce qu'elle peut être l'agent par excellence de Satan.

\section{Quelle image le discours officiel projette-t-il de la femme?}

Le moindre défaut de la femme, c'est d'être bavarde, car le silence est d'or. Mais, en outre, c'est la femme qui risque d'entraîner l'homme hors de sa vocation. C'est la femme qui risque de l'entraîner au péché de luxure, qui est le péché redouté par excellence par les hommes d'Église, bien que ce ne soit pas celui qui soit en tête des péchés capitaux. C'est par la femme que tous les malheurs peuvent arriver, le péché de luxure et puis la brouille dans les ménages, la discorde dans le foyer. Donc, elle est vraiment culpabilisée au maximum. Au XIVe siècle un religieux, qui était bien en cour auprès des papes d'Avignon, a dressé une liste (que j'ai reproduite dans mon livre) des cent deux défauts de la femme. Cela évidemment nous fait rire, avec raison. Mais ce livre a été écrit et il explique, entre autres, la multiplication postérieure des ouvrages de démonologie et il fait aussi comprendre qu'à l'époque où les procès de sorcellerie se sont multipliés, c'est-à-dire aux XVIe et XVIIe siècles, on a plus condamné de femmes que d'hommes. La femme était réputée plus fragile que l'homme. Elle était aussi plus facilement convaincue par Satan et devenait plus facilement sorcière que l'homme.

La grande répression contre la sorcellerie est-elle la réponse des autorités à l'omniprésence de la peur?

Sûrement! À cet égard je dois tout de même apporter une précision. On a beaucoup travaillé sur la question de la sorcellerie durant les trente dernières années. On a ouvert des dossiers qui ont été très bien étudiés par tous nos collègues historiens. Moi-même je n'ai pas apporté de nouvelles révélations sur les procès de sorcellerie. En revanche, dans mon livre, j'ai essayé, et je crois que j'ai été le seul, de replacer la peur de la sorcellerie à l'intérieur d'un ensemble de peurs. Autrement dit, la répression de la sorcellerie ne se comprend pas si on l'isole de tout un contexte et c'est ce contexte de peur globale que j'ai essayé de remettre en lumière et à l'intérieur duquel s'est située précisément la répression de la sorcellerie. On a réprimé la sorcellerie comme on réprimait les blasphémateurs et comme on luttait contre les hérétiques ${ }^{7} \cdot[\ldots]$

\section{TRANSGRESSIONS?}

Si l'on regarde Scène de village de Bruegel, par exemple, peut-on en déduire que, dans la vie quotidienne, les Européens n'observaient pas vraiment les enseignements de l'Église?

Pour Bruegel, vous pensez aux scènes de festin? [...] Les scènes de festin, il en faut. Mais les banquets conduisaient sûrement à des excès, à des péchés. Alors on rejoint ce que j'ai écrit dans mon livre L'aveu et le pardon, brève histoire de la confession au pays catholique. Comme la confession était une contrainte sérieuse, les confesseurs ont été, à mon avis, extrêmement indulgents jusqu'à la réaction rigoriste du XVIIe siècle. Auparavant, je dirais qu'on accordait le pardon facilement. De sorte que les pécheurs qu'étaient, comme nous, nos ancêtres avaient conscience de commettre des péchés, mais avaient aussi la conviction qu'ils étaient facilement pardonnés. Les choses en revanche se sont dramatisées à partir de la réaction rigoriste, à l'époque d'Arnauld, Pascal, etc. Donc pour répondre complètement à votre question, je pense que les gens des XVe et XVIe siècles avaient le sentiment d'être chrétiens et à la fois d'être pécheurs et pardonnés. [...]

L'insécurité religieuse est étroitement associée à l'idée de péché, mais ce sentiment était-il partagé par l'ensemble des croyants?

Je pense qu'il était assez largement partagé, parce que venant, certes, de l'élite il a constitué une des composantes de la mentalité occidentale. Et par là, on rejoint saint Augustin. Pour celui-ci, la vie est un combat. Nous sommes dans la cité terrestre, en route vers la cité céleste et pour parvenir à cette dernière nous devons combattre. La vie est une lutte perpétuelle contre le démon, contre tous ses prestiges et sortilèges. Il faut donc être en alerte. C'est le sens de la formule de Luther: "Arrière tous ceux qui disent: paix!» Phrase typiquement augustinienne. La paix, c'est pour l'au-delà; maintenant il nous faut combattre. "Ne nous endormons pas - c'est encore une phrase de Luther - ne nous endormons pas sur l'oreiller d'une fausse sécurité.»

L'homme occidental, j'élargis le propos, a été un homme en état d'alerte, invité à faire son examen de conscience et à se critiquer lui-même. Pas pour une introspection stérile, pas pour se complaire dans la morosité, mais pour progresser. Je crois que, si l'on considère ce qu'a été l'histoire de la civilisation en Occident, on peut dire qu'elle a été l'histoire d'initiatives continuellement renouvelées, celle d'un homme perpétuellement actif. L'homme occidental a, certes, ses défauts, mais il est par excellence l'homme qui ne se repose pas. C'est typiquement augustinien.

\section{PRATIQUES SÉCURITAIRES}

À l'encontre des prédicateurs qui tenaient un discours plutôt pessimiste, vous citez le philosophe Spinoza: "Quand on est sûr d'atteindre au bien supposé, l'âme acquiert ce repos que nous nommons sécurité." Spinoza est-il l'exception à la règle?

Spinoza se situe en dehors d'un discours chrétien et, d'autre part, dans ses propos éthiques ou philosophiques, il n'a pas vraiment cerné le concept de sécurité, tel que je l'ai étudié dans mon livre Rassurer et protéger. En revanche, je dirais que Spinoza est plus percutant et plus convaincant lorsqu'il parle de la sécurité sur le plan civique et politique. Et à ce moment-là, il rejoint Machiavel, Hobbes et même Bossuet. Car ils pensent en commun que le but de la cité est d'assurer la sécurité des citoyens ${ }^{8}$. Ce qui fait qu'il y a eu deux discours différents sur la sécurité: le discours éthique et chrétien qui faisait l'éloge de l'insécurité, parce qu'il ne faut pas s'endormir dans la fausse sécurité quand il s'agit du salut, et le discours politique, selon lequel la société doit assurer la sécurité des citoyens. Le mot "sécurité" prend alors une connotation totalement positive, alors que, dans le discours théologique, il avait plutôt une connotation négative. [...] 
LA COMMUNION DES SAINTS POSTULE QU'IL Y A INTERACTION ENTRE LES VIVANTS

ET LES DÉFUNTS.

Pour s'assurer le repos éternel, l'Église a élaboré certaines pratiques dont la pratique de la vente des indulgences. À ce propos vous citez le cardinal Bellarmin, qui écrit en 1599: "C'est une chose très certaine et hors de doute chez les catholiques que les âmes qui souffrent au purgatoire peuvent être aidées par les indulgences." Comment s'est formée cette idée?

Je vais faire un correctif: à l'époque de Bellarmin on ne vendait plus d'indulgences. Le concile de Trente a totalement arrêté cette pratique qui n'a existé que durant la période antérieure au concile de Trente. Mais au temps de Luther, ce fut la goutte d'eau qui fit déborder le vase.

La doctrine des indulgences se fonde sur une conception chrétienne qui est très belle, à mon avis, celle de la "communion des saints" - j'emploie l'expression classique. La communion des saints postule qu'il y a interaction entre les vivants et les défunts. Ils ne sont pas séparés par la mort, mais continuent d'agir, en quelque sorte, les uns pour les autres, à condition naturellement que les trépassés soient sauvés - on ne parle pas de ceux qui sont tombés en enfer. Mais les âmes du purgatoire, en bonne théologie catholique, peuvent prier pour les vivants; et de même les vivants pour les âmes du purgatoire. Il y a donc réciprocité. Je crois profondément, pour ma part, à la communion des saints, c'est-àdire à cette continuité des rapports entre les morts et les vivants. Ce qui ne veut pas dire que je crois aux revenants - ça, c'est une autre question!

La pratique des indulgences s'est créée à partir de cette doctrine et aussi à partir de ce qu'on appelait le "trésor de l'Église», c'est-à-dire les mérites du Christ et des saints. L'Église et les prières des fidèles pouvaient puiser dans ce "trésor» et donc obtenir des indulgences ou si vous voulez des raccourcissements du purgatoire pour les morts. Voilà comment les choses se sont produites. Mais le système a dérivé à partir du moment où il y a eu vente. Naturellement, il faut s'expliquer sur le mot «vente». On ne vendait pas comme ça les indulgences. Il fallait s'être confessé et avoir communié; en plus on donnait une aumône. Plus l'aumône était forte, évidemment plus on était censé obtenir d'indulgences pour soi ou pour les autres. À cette dérive s'en est ajoutée une autre: car on se mit à compter le temps de purgatoire avec nos horloges en jours, mois et années. Alors à ce moment-là, c'était la dérive complète. On pouvait, tel jour, en faisant telle prière, obtenir 500, 1000 ans ou je ne sais combien de rabais de purgatoire. On entrait dans ce qu'un de nos collègues Jacques Chifollau a appelé la "comptabilité de l'au-delà» et on frisait l'absurde.

\section{Ce sont les abus que Luther a dénoncés?}

Bien sûr. Mais, il a surtout dénoncé la vente des indulgences, qui a été, je répète, arrêtée par le concile de Trente.
Était-ce à ce point lucratif pour l'Église?

Dans un petit livre intitulé Le cas Luther, j'ai rappelé qu'il s'agissait d'indulgences qui étaient vendues, notamment en Allemagne, pour que le produit financier aide à la reconstruction de la basilique Saint-Pierre. Mais je ne pense pas que cela ait rapporté des sommes très considérables. Néanmoins, l'apport n'a pas été nul et il fallait que les sommes soient rassemblées quelque part. C'est la banque Fugger qui s'en chargeait en Allemagne. Alors, à ce moment-là, on entrait dans un circuit financier qui, évidemment, éloignait beaucoup des intentions purement spirituelles...

\section{Et du salut éternel...}

... et du salut éternel!

Saint Joseph, à partir du XVIIe siècle, devient l'objet d'un véritable culte au Canada français. Saint Joseph occupe une place particulière.

Notamment sa basilique qui domine Montréal, j'y suis allé.

En quoi saint Joseph pouvait-il être rassurant à l'article de la mort?

Tout d'abord il faut dire que l'image de saint Joseph a complètement changé à partir du XVIe siècle. Car si vous regardez des tableaux du XVe siècle et encore du tout début du XVIe siècle, qui représentent la nativité, vous y voyez saint Joseph presque toujours représenté comme un vieillard: ou bien il dort au coin du feu ou bien il prépare la bouillie. Souvent c'est un vieillard trop âgé pour avoir des enfants.

Or, cette image disparaît à partir du XVIe siècle et saint Joseph devient un homme jeune et actif, vraiment le père adoptif de Jésus. En même temps, à partir des années 1520, a été diffusée à partir de l'Italie, une vie de saint Joseph, fondée sur les apocryphes et non sur les Évangiles, où l'on évoquait la mort de saint Joseph. Cette mort était racontée de façon très émouvante, Joseph s'endormant dans la mort et dans les bras de Jésus. Si quelqu'un a eu une bonne mort, c'est bien saint Joseph. Il faut dire qu'il la méritait. En tout cas, on a cru, à partir de cet ouvrage, que saint Joseph avait eu la mort idéale. On l'a donc tout naturellement invoqué comme patron de la bonne mort. Il est devenu l'être sécurisant par excellence dans le moment du dur passage où l'on doit se déprendre de tout. C'est à ce titre qu'i1 a été le plus souvent invoqué ensuite dans l'histoire occidentale.

Croyait-on vraiment que la pratique du rosaire et que les scapulaires pouvaient attirer les grâces divines?

Je le crois. Je suis persuadé que ceux qui récitaient leur rosaire ou qui portaient des scapulaires croyaient avoir ainsi une protection contre les malheurs d'ici-bas et les dangers de l'au-delà. Mais ce résultat n'était pas attendu du seul fait de porter un scapulaire ou de réciter machinalement un rosaire. Car on priait en même temps qu'on portait le scapulaire ou qu'on récitait le rosaire.

Au-delà des inévitables routines et distractions, il y avait une vraie prière. En outre, le port du scapulaire était accompagné d'un certain nombre de pratiques religieuses. On ne portait pas seulement le scapulaire! Il y avait tout un cheminement religieux qui s'y rattachait. À mon avis, le chapelet et le scapulaire ont eu réellement des fonctions religieuses de sécurisation. 


\section{SÉCURISATION, TOLÉRANCE ET DISPOSITION AU BONHEUR}

Au XVIIIe siècle, le sentiment d'insécurité semble diminuer. Vous citez de nombreux auteurs tels que JeanJacques Rousseau qui dénoncent la "pastorale de la peum" et demandent à l'Église de rassurer les croyants. Pourquoi ce changement?

Dans mon livre Rassurer et protéger, j'ai marqué la concomitance entre une diminution de l'insécurité dans la vie quotidienne en Occident et l'émergence d'un discours hostile à la pastorale de la peur dans l'Église. Je ne sais pas s'il y a vraiment eu relation de cause à effet, mais je constate historiquement que les deux phénomènes se sont produits en même temps. Mes lecteurs en tireront la conclusion qu'ils voudront.

À cet égard, j'ai fait, si j'ose dire, l'apologie de la philosophie des Lumières et je crois en effet que notre monde occidental actuel est tributaire à la fois de l'héritage chrétien et de l'héritage des Lumières. Je ne veux donc pas excommunier l'héritage des Lumières, car je crois que les gens des Lumières ont eu le mérite - je parle en chrétien, mais en historien chrétien - de montrer à l'Église qu'elle se trompait de discours sur Dieu. Ce faisant, les hommes des Lumières ont provoqué ce que j'ai appelé «une réhabilitation de Dieu». [...]

Peut-on dire que la déchristianisation résulte du discours de la peur?

Je crois effectivement que l'image répulsive qu'on avait présentée de Dieu a été, non pas la seule, mais l'une des causes de la déchristianisation. [...]

\section{Avec le développement de la tolérance aux XVIle et XVIIle siècles, la peur se dissipe-t-elle?}

Elle diminue, je ne dis pas qu'elle disparaît parce que la peur ne disparaîtra pas, la peur est fondamentale chez l'homme et tant qu'il y aura des hommes, ils auront peur. Mais on peut davantage dominer ses peurs. Ce que j'ai essayé de montrer, c'est que toutes ces peurs, dont j'avais remarqué qu'elles étaient liées entre elles, se sont exténuées en même temps. Ce n'est pas par hasard qu'à partir de 1650 diminue la lutte contre les hérétiques et s'essoufflent les procès de sorcières en même temps, d'ailleurs, que diminue aussi la lutte contre les blasphémateurs. Quant au Turc, il a fini d'avancer. Il s'est donc produit un essoufflement des peurs que j'avais étudiées dans mon livre.

\section{Et peut-on parler d'un essor de l'idée de bonheur?}

Oui, le XVIIIe siècle voit naître l'idée que la terre pourrait ne pas être seulement une "vallée de larmes». Cela, c'était une idée neuve. Et c'est important!9

\section{Notes}

1 Extraits tirés de l'ouvrage Les grandes controverses de l'histoire, Sillery (Québec), Septentrion et Société RadioCanada, 1996, 207 pages. La rédaction, qui a réaménagé l'ordre des sections et des paragraphes, remercie les auteurs ainsi que les Éditions Septentrion pour leur aimable autorisation.

2 Voir Jean DELUMEAU, Rassurer et protéger. Le sentiment de sécurité dans l'Occident d'autrefois, Paris, A. Fayard, 1989, 667 pages.

3 N.D.L.R.: on observe également ces croyances populaires au Québec, à travers les données ethnographiques, les légendes et les contes, voire les survivances chuchotées.

4 Voir Jean DELUMEAU, Le péché et la peur. La culpabilisation en Occident (XIIIe-XVIIIe siècles), Paris, Fayard, 1983; Guy BECHTEL, La chair, le diable et le confesseur, Paris, Plon, coll. "Le doigt de Dieu», 1994.

Norman COHN, Les fanatiques de l'Apocalypse, Paris, Payot, 1983.

5 N.D.L.R.: on le constate, les prophéties apocalyptiques n'ont pas trait qu'au passage de l'an mil: pour ce qui concerne spécifiquement le second schéma dont parle Jean Delumeau, sur la fin du monde associée au premier millénaire, voir infra Que trouver sous les zéros? sur les travaux de Georges DUBY

Cela dit, précisons ceci: cette "allégorie spirituelle» (saint Augustin), née de la chrétienté, le "millénarisme» ou l'attente anxieuse de catastrophes auxquelles succéderait un monde nouveau, une sorte d'âge d'or de l'humanité, a pris des formes variées. À toutes les époques, cette croyance a servi de véhicule aux aspirations comme aux animosités sociales, parfois même dans le délire. Ne serait-ce que dans l'Occident contemporain, on peut recenser les mille ans de bonheur prédits par Hitler, le messianisme marxiste qui a créé les goulags, les propagandes sectaires...

6 N.D.L.R.: à propos d'Ève, non plus comme cristallisation du féminin pécheur, mais symbole d'une humanité blessée, voir Annick de SOUZENELLE, Le symbolisme du corps humain. De l'arbre de la vie au schéma corporel, St-Jean-de Brayes, Éditions Dangles, 1984. Sur les peurs ataviques à propos des femmes, voir Frontières, «Le féminin et la mort», vol. 7, no 1, printemps 1994.

7 Voir Jean DELUMEAU, La peur en Occident, Paris, Fayard, 1978; Carlo GINZBURG, Le sabbat des sorcières, Paris, Gallimard, 1989; Henry INSTITORIS et Jacques SPRENGER, Le marteau des sorcières. Malleus Maleficarum, Paris, Jérôme Millon, 1990; Brian P. LEVACK, La grande chasse aux sorcières en Europe aux débuts des temps modernes, Paris, Champs Vallon, coll. "Époques», 1987.

8 N.D.L.R.: voir l'article de Wilfrid Noël RABY dans ce numéro.

9 N.D.L.R.: l'articulation des peurs qui ressort de l'ouvrage La Peur en Occident est éclairante pour l'actualité. Ainsi Jean DELUMEAU distingue:

1- Peurs spontanées, construites en lien avec le réel: a) peurs universelles, "naturelles" (la nuit); b) peurs liées à un niveau technique insuffisant et à l'outillage mental qui lui correspond (même de nos jours); c): peurs cycliques, bouleversant les catégories de participation populaire (crises économiques, guerres, épidémies); d) peurs existentielles, différentes selon les époques (vieillir, utilité sociale)

2- Peurs spontanées, construites sans lien avec le réel: (peur du purgatoire et de l'enfer)

3- Peurs organisées, réfléchies: (par gradation) peurs objectivées par ceux qui ont peur, peurs conduites par les «directeurs de conscience" du temps, assurant leur domination; peurs délirantes individuelles, mais sous cette rubrique, surtout collectives 